\title{
Análisis térmico, modelamiento matemático y simulación de un reactor de agitación discontinuo para volumen específico
}

\section{Thermal analysis, mathematical modeling and simulation of a discontinuous agitation reactor for specific volume}

\author{
Humberto Rolón-Ortiz ${ }^{1 a}$, Carlos Acevedo-Peñaloza², Yesenia Villamizar-González ${ }^{1 b}$ \\ ${ }^{1}$ Grupo de Investigación de Fluidos y Térmicas (Fluter), programa de Ingeniería Mecánica, Universidad Francisco de Paula \\ Santander, Colombia. Correos electrónicos: ${ }^{\mathrm{a}}$ humbertoalejandroro@ufps.edu.co, ${ }^{\mathrm{b}}$ yeseniavg@ufps.edu.co \\ ${ }^{2}$ Grupo de Investigación en Diseño Mecánico, Materiales y Procesos (Gidima), programa de Ingeniería Mecánica, Universidad \\ Francisco de Paula Santander, Colombia. Correo electrónico: carloshumbertoap@ufps.edu.co \\ Recibido: 14 noviembre, 2017. Aceptado: 3 mayo, 2018. Versión final: 19 junio, 2018.
}

\begin{abstract}
Resumen
El presente trabajo expone el análisis térmico y el modelamiento matemático de un reactor de agitación discontinuo. Se implementó una fase de adquisición de datos en cuanto al funcionamiento y la utilidad de los reactores de agitación. Posteriormente, se aplicaron los conceptos de termodinámica, mecánica de fluidos, transferencia de calor y transporte de materia, para realizar el respectivo análisis térmico, y así determinar la energía contenida y transferida en los fluidos utilizados. Por último, se dedujo un modelo matemático en el software EES, para comprobar su comportamiento, teniendo en cuenta las variables operativas sometidas a diversos tipos de fluidos. Además, se simuló el entorno físicoquímico del reactor, para validar los datos calculados y compararlos con los valores experimentales, relacionando la geometría del equipo con las condiciones de trabajo y las especificaciones del producto final.
\end{abstract}

Palabras clave: mecánica de fluidos; reactor de agitación; termodinámica; transferencia de calor.

\begin{abstract}
This paper presents the thermal analysis and mathematical modeling of a discontinuous agitation reactor. A data acquisition phase was implemented in terms of operation and utility of the agitation reactors. Subsequently, the concepts of thermodynamics, fluid mechanics, heat transfer and matter transport were applied to perform the respective thermal analysis and thus, determine the energy contained and transferred in the fluids used. In addition, a mathematical model was deduced in the EES software to examine its behavior, taking into account the operational variables subjected to various types of fluids. Finally, the physical-chemical environment of the reactor was simulated to validate the calculated data and compare them with the experimental values, relating the geometry of the equipment to the performance conditions and the specifications of the final product.
\end{abstract}

Keywords: fluid mechanics; heat transfer; stirred reactor; thermodynamics.

\section{Introducción}

\subsection{Reactores químicos}

Un reactor químico es una unidad procesadora, diseñada para que en él se lleven a cabo una o varias reacciones químicas. Esta unidad procesadora está constituida por un recipiente cerrado, que cuenta con líneas de entrada y salida de sustancias químicas. Se pretende diseñar un reactor con el objetivo de maximizar la conversión y la selectividad de una reacción con el menor coste posible. Es por esto que la idea de realizar este proyecto surge de

ISSN impreso: 1657 - 4583, ISSN en línea: 2145 - 8456, CC BY-ND 4.0 (c) (i) $९$

Como citar: H. Rolón-Ortiz, C. Acevedo-Peñaloza, Y. Villamizar-González, "Análisis térmico, modelamiento matemático y simulación de un reactor de agitación discontinuo para volumen específico," Rev. UIS Ing., vol. 18, no. 1, pp. 39-48, 2019. doi: 10.18273/revuin.v18n1-2019003 
la necesidad de crear un combustible con componentes biológicos, que permitan disminuir los efectos adversos de la emisión de gases de invernadero.

Para producir este tipo de biocombustibles, se emplea el método de transesterificación, que se fundamenta en la reacción de un triéster de glicerilo (triglicérido) con un alcohol, para formar alquil ésteres y glicerol [1]. En pocas palabras, consiste en mezclar diversos fluidos a una velocidad y una temperatura determinadas, para lograr un proceso químico de excelente calidad, reduciendo los costos de operación al mínimo.

La reacción de transesterificación puede llevarse a cabo por diferentes vías. La más común de ellas es utilizar reactores de tipo Bach. Existen diversas investigaciones realizadas con base en este tipo de reactores, y en ellas se evalúan las diferentes condiciones de transesterificación [2], [3], [4]. Las variables típicas de reacción incluyen una relación molar de 6:1 metanol: aceite, agitación vigorosa, temperatura de $60{ }^{\circ} \mathrm{C}$ a $90{ }^{\circ} \mathrm{C}$ a presión atmosférica y la cantidad de catalizador ronda el $1 \%$, en peso, de la masa total de la fuente oleosa. Otra vía de transesterificación es la utilizar reactores continuos mediante catálisis heterogénea, opción que en general resulta más costosa, debido a la fuente de síntesis del catalizador como reactor, además de que requiere largos periodos de reacción [5].

Los reactores químicos tienen las siguientes funciones principales: asegurar el tipo de contacto (modo de fluir) de los reactantes dentro del tanque, para conseguir una mezcla deseada con los materiales reactantes; proporcionar el tiempo suficiente de contacto entre las sustancias y el catalizador, para conseguir la extensión deseada de la reacción, y permitir condiciones de presión, temperatura y composición, de modo que la reacción tenga lugar en el grado y la velocidad deseada, atendiendo a los aspectos termodinámicos y cinéticos de la reacción [6].

En el presente trabajo se han realizado ensayos en un reactor de agitación con las adecuaciones necesarias para convertirlo en un proceso discontinuo. Enseguida se presentan las características físico-químicas de este tipo de sistemas.

\subsection{Tipos de reactores}

Los más conocidos son los reactores discontinuos, en donde los reactantes se introducen en el reactor, se mezclan, se deja que reaccionen en un tiempo determinado y finalmente se descarga la mezcla resultante. Esta es una operación no estacionaria en la que la composición va variando con el tiempo, manteniendo la uniformidad en todos los puntos del reactor.

El primero de los dos reactores ideales de flujo estacionario es el reactor de flujo en pistón. Este reactor se caracteriza porque el flujo es perpendicular a una sección transversal. Asimismo, es ordenado; ningún elemento de este se sobrepasa o se mezcla con cualquier otro elemento situado antes o después de él; en realidad, en este reactor puede haber mezcla lateral de fluido, pero nunca ha de existir mezcla o difusión a lo largo de la trayectoria de flujo. La condición necesaria y suficiente para que exista flujo en pistón es que el tiempo de residencia en el reactor sea el mismo para todos los elementos del fluido [7].

El otro reactor ideal de flujo estacionario, se denomina reactor de mezcla completa. Como su nombre lo indica, su contenido está perfectamente agitado y su composición en cada instante del proceso es la misma a lo largo del reactor. Por consiguiente, la corriente de salida de este reactor tiene la misma composición que la del fluido contenido en el mismo [8].

\subsection{Procesos continuos y discontinuos}

Un proceso discontinuo, o por lotes, se da cuando los reactivos son precargados y se vacían cuando la reacción se ha completado.

La ventaja clave de este tipo de procesos es la flexibilidad. Por eso se usan principalmente en la industria química, alimentaria o farmacéutica, donde es muy importante ofrecer una gran variedad de productos e introducir otros nuevos rápidamente, ya que estos sectores industriales operan en mercados altamente competitivos.

Los equipos de procesos discontinuos se diseñan para manejar un amplio rango de operaciones y productos. La flexibilidad permite manufacturar nuevos productos sin tener que construir una nueva planta o sin tener que hacer grandes cambios de equipos. Esta flexibilidad también permite producir pequeñas cantidades de un producto sin arriesgar toda la productividad [9]. En los procesos discontinuos una desviación excesiva de la reacción, suele ser difícil de controlar. Por ello, frecuentemente para reacciones exotérmicas y para las de dos fases (gaslíquido), se utiliza una operación semidiscontinua. En estas, solo una parte de los reactivos son cargados inicialmente y el resto son añadidos de forma controlada. De este modo, si ocurre una pérdida de control es posible detener la carga de reactivos. Los procesos por lotes (discontinuos) se caracterizan por trabajar en condiciones no estacionarias, es decir que durante la operación tanto la composición como la generación de calor cambian [9]. 
específico

Por otro lado, los procesos continuos se suelen realizar en recipientes agitados, ya sea de modo simple o en cascada, con tuberías de flujo de descarga. En estos sistemas todos los reactivos son continuamente cargados al reactor, y los productos son continuamente descargados. Los procesos continuos se caracterizan por trabajar en condiciones estacionarias, en las que tanto el calor generado como la composición química permanecen constantes durante toda la operación.

Los procesos continuos tienen la ventaja de producir en gran volumen un producto estándar que generalmente proporciona una buena recuperación del capital invertido. Dado que los requerimientos de productos no cambian significativamente el proceso necesitará mínimas modificaciones durante su vida de trabajo para mantenerse competitivo [10].

En la tabla 1 se aprecia una comparación entre los procesos continuos y discontinuos, que evalúa diferentes características y variables de producción.

Tabla 1. Comparación entre procesos discontinuos y continuos

\begin{tabular}{|c|c|c|}
\hline Características & Discontinuo & Continuo \\
\hline $\begin{array}{c}\text { Operación de } \\
\text { procesos }\end{array}$ & $\begin{array}{l}\text { Ocurre una } \\
\text { secuencia } \\
\text { específica }\end{array}$ & $\begin{array}{l}\text { Es continuo, } \\
\text { simultáneo }\end{array}$ \\
\hline $\begin{array}{c}\text { Diseño de } \\
\text { equipos, uso }\end{array}$ & $\begin{array}{c}\text { Diseñado para } \\
\text { obtener muchos } \\
\text { productos }\end{array}$ & $\begin{array}{c}\text { Diseñado para } \\
\text { obtener productos } \\
\text { específicos }\end{array}$ \\
\hline Producto & Cantidad limitada & Flujo continuo \\
\hline Entorno & $\begin{array}{c}\text { Variable, a } \\
\text { menudo cambia } \\
\text { notablemente entre } \\
\text { operaciones }\end{array}$ & $\begin{array}{l}\text { Usualmente, } \\
\text { estado fijo con } \\
\text { presión, flujo y } \\
\text { cantidades } \\
\text { constantes } \\
\end{array}$ \\
\hline $\begin{array}{c}\text { Intervención del } \\
\text { operador }\end{array}$ & $\begin{array}{c}\text { Necesario, } \\
\text { regularmente } \\
\text { como parte de las } \\
\text { operaciones de } \\
\text { trabajo }\end{array}$ & $\begin{array}{l}\text { Principalmente, } \\
\text { para corregir } \\
\text { condiciones } \\
\text { anormales }\end{array}$ \\
\hline
\end{tabular}

Fuente: elaboración propia.

\section{Metodología}

\subsection{Factores de diseño}

Para elegir un reactor, es conveniente realizar un balance técnico-económico completo, teniendo en cuenta elementos como el tipo de operación y el mantenimiento a realizar, además se debe elegir la configuración más apropiada que dependerá principalmente de los factores físico-químicos propios de cada tipo de reacción.
El diseño de reactores tiene como fin seleccionar los elementos técnicos, mecánicos y químicos más apropiados, y ensamblarlos de forma que estos operen eficientemente. Por esto es necesario conocer tanto la cinética como la termodinámica de las reacciones de interés. Otras disciplinas importantes para el diseño de reactores son la mecánica de fluidos, la transferencia de materia y la transmisión de calor [11].

Para el diseño de un reactor se debe tener en cuenta que las condiciones en este varían con la posición y con el tiempo, para ello es necesario efectuar la integración adecuada de la ecuación cinética para las condiciones de operación. En la tabla 2 se comparan los diferentes tipos de reactores de acuerdo con el régimen de operación y según los principios físico-químicos, al tiempo que se resaltan las ventajas y las desventajas propias de cada uno.

Tabla 2. Comparación entre diferentes tipos de reactores

\begin{tabular}{|c|c|c|}
\hline $\begin{array}{l}\text { Tipo de } \\
\text { reactor }\end{array}$ & Ventajas & Desventajas \\
\hline $\begin{array}{c}\text { PFR (reactor } \\
\text { de flujo } \\
\text { pistón) }\end{array}$ & $\begin{array}{c}\text { Bajo inventario. } \\
\text { Condiciones } \\
\text { estacionarias. }\end{array}$ & $\begin{array}{l}\text { Dependencia del } \\
\text { proceso. } \\
\text { Posibles puntos } \\
\text { calientes. } \\
\text { Agitación presente } \\
\text { solo en mezclas en } \\
\text { línea. } \\
\text { Difícil de diseñar. }\end{array}$ \\
\hline $\begin{array}{l}\text { Reactor } \\
\text { continuo de } \\
\text { mezcla } \\
\text { completa }\end{array}$ & $\begin{array}{l}\text { Condiciones } \\
\text { estacionarias. } \\
\text { Presenta } \\
\text { herramienta de } \\
\text { seguridad. } \\
\text { El flujo puede } \\
\text { ser diluido para } \\
\text { ralentizar la } \\
\text { reacción. } \\
\end{array}$ & $\begin{array}{l}\text { Gran inventario. } \\
\text { Difícil de enfriar } \\
\text { grandes masas. } \\
\text { Problemas de } \\
\text { precipitación. } \\
\text { Bajo rendimiento } \\
\text { global. }\end{array}$ \\
\hline $\begin{array}{c}\text { Reactor } \\
\text { discontinuo }\end{array}$ & $\begin{array}{c}\text { Presenta } \\
\text { herramienta de } \\
\text { seguridad. }\end{array}$ & $\begin{array}{c}\text { Reacciones } \\
\text { exotérmicas difíciles } \\
\text { de controlar. } \\
\text { Grandes inventarios. } \\
\text { Todos los materiales } \\
\text { presentes en el } \\
\text { proceso. } \\
\end{array}$ \\
\hline $\begin{array}{l}\text { Reactor } \\
\text { semidiscon- } \\
\text { tinuo }\end{array}$ & $\begin{array}{l}\text { Velocidad de } \\
\text { adición } \\
\text { controlable. } \\
\text { Reacción } \\
\text { exotérmica } \\
\text { controlable. } \\
\end{array}$ & $\begin{array}{c}\text { Temperatura de inicio } \\
\text { crítica (si es muy } \\
\text { baja, se acumulan } \\
\text { reactivos). } \\
\text { Problemas de } \\
\text { precipitación. } \\
\end{array}$ \\
\hline
\end{tabular}

Fuente: elaboración propia. 
El punto de partida para el diseño de un reactor es un balance de materia propio del reactante (o producto) a utilizar, sobre determinado volumen de control.

$$
\begin{aligned}
\left(\begin{array}{c}
\text { entrada de } \\
\text { reactante al } \\
\text { volumen de } \\
\text { control }
\end{array}\right)= & \left(\begin{array}{c}
\text { salida de } \\
\text { reactante de } \\
\text { volumen del } \\
\text { control }
\end{array}\right)+\left(\begin{array}{c}
\text { desaparición } \\
\text { de reactante } \\
\text { por reacción } \\
\text { química }
\end{array}\right) \\
& +\left(\begin{array}{c}
\text { acumulación } \\
\text { de reactante } \\
\text { en el volumen } \\
\text { de control }
\end{array}\right)
\end{aligned}
$$

En las operaciones no isotérmicas debe agregarse también el balance de energía, que está relacionado con el término de reacción química, ya que el calor generado o absorbido es proporcional a la extensión de la reacción. No obstante, para el presente trabajo estos aspectos no serán tomados en consideración, y se asumirá que se trabaja en condiciones isotérmicas [11].

El reactor del caso de estudio es de tipo tanque agitado, envuelto por una camisa exterior que permite el calentamiento o enfriamiento de la masa reaccionante, según convenga (figura 1). Este tipo de reactores es ampliamente utilizado en industrias con producciones pequeñas, en las que el coste de operación y el tiempo muerto representan solamente una pequeña fracción del coste unitario del producto. Es por eso que abastecen el mercado de los bienes de alto valor añadido como los correspondientes a la industria de la química fina, específicamente en plantas en las que se necesita un completo portafolio de productos.

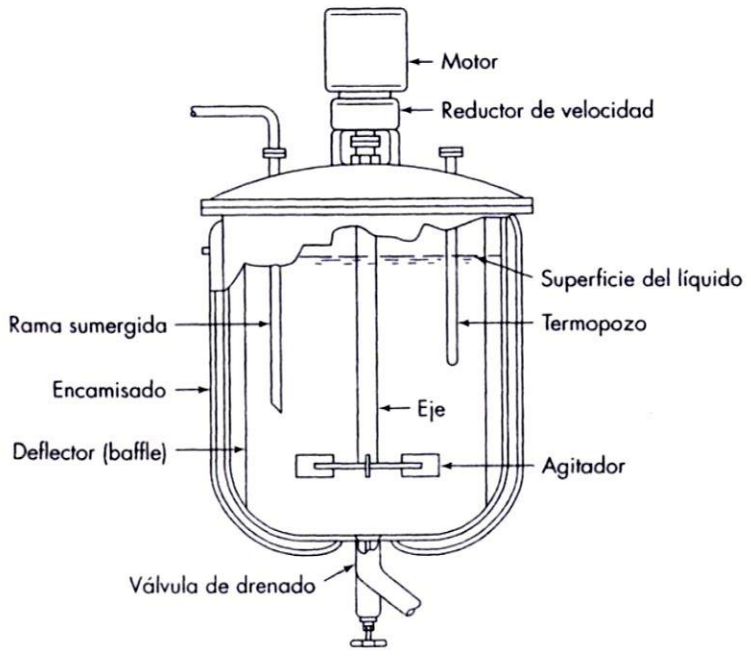

Figura 1. Tanque típico del proceso de agitación. Fuente: Denbigh y Turner, 1990.

De esta forma, se selecciona el tipo de reactor apropiado para las pruebas matemáticas y la simulación. El dispositivo adecuado es un reactor de agitación discontinuo (figura 2), que cuenta con una capacidad de almacenaje de 10 litros, está reforzado con camisa de acero y posee un serpentín como herramienta de disipación de calor en aluminio.

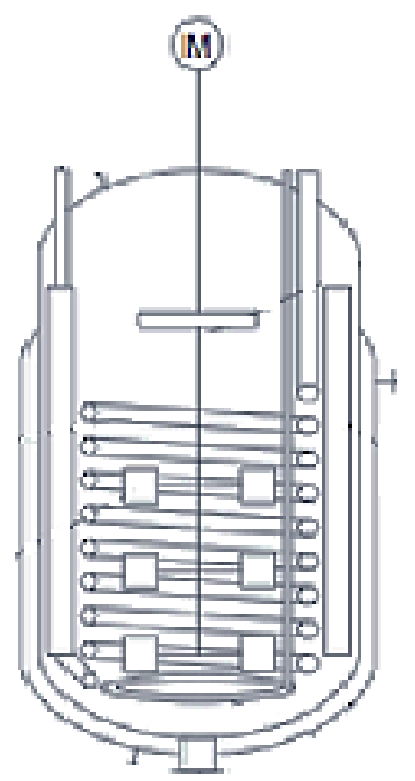

Figura 2. Reactor de agitación discontinuo. Fuente: Towler y Sinnott, 2013.

\subsection{Características del cilindro para un volumen de $10 \mathrm{~L}$}

A continuación, en la figura 3, se muestra la discretización de un volumen de control de $10 \mathrm{~L}$, el cual hace parte del caso de estudio.

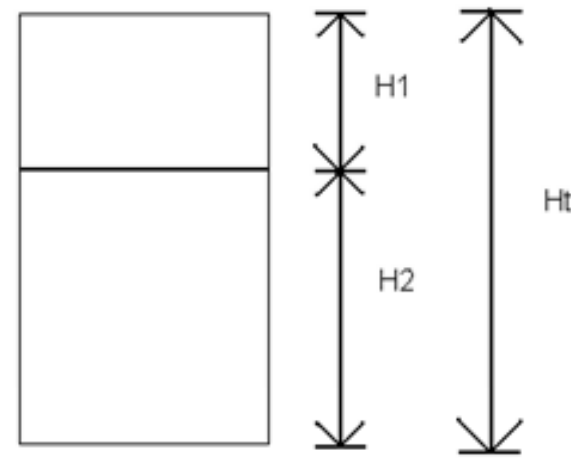

Figura 3. Volumen de control. Fuente: elaboración propia.

Se escriben las distintas expresiones matemáticas que involucran las correlaciones de volumen de trabajo de acuerdo con la cantidad de producto final que se quiere obtener. Las variables incluyen el área de mezclado, de transferencia y de disipación de calor [12]. 
Análisis térmico, modelamiento matemático y simulación de un reactor de agitación discontinuo para volumen específico

$$
\begin{gathered}
V t=V 1+V 2 \\
V 2=A * H 2 ; \quad V 2=10 l=0.01 \mathrm{~m}^{3} \\
0.01 \mathrm{~m}^{3}=\frac{\pi}{4} *(0.2 \mathrm{~m})^{2} * H 2 \\
H 2=0.32 \mathrm{~m}=32 \mathrm{~cm}
\end{gathered}
$$

Ahora, teniendo en cuenta un factor de seguridad (Fs) de 0.25 , en referencia a la altura anterior para el volumen inicial de $10 \mathrm{~L}$, se obtiene que $\mathrm{H} 1=8 \mathrm{~cm}$. Dicho lo anterior $H t=8 \mathrm{~cm}+32 \mathrm{~cm}=40 \mathrm{~cm}$.

Entonces:

$$
\begin{gathered}
V t=0.01 m^{3}+\frac{\pi}{4} *(0.2 m)^{2} * 0.08 m=0.0125 m^{3} \\
V t=10.125 l
\end{gathered}
$$

También se analiza la longitud de tubo del reactor, que se basa en la cantidad de espirales que rodean el sistema de almacenaje de reactivos y catalizadores. Según esto, se tiene lo siguiente:

Número de espiras: 6,

Espacio entre espiras: $2.5 \mathrm{~cm}$

$$
\begin{gathered}
d_{\text {medio intercambiador }}=14.8 \mathrm{~cm} \\
\text { Ltubo }=\left(N^{\circ} \text { espiras } * \pi * d\right)+4 \mathrm{~cm} \\
\text { Ltubo }=(6 * \pi * 14.8 \mathrm{~cm})+4 \mathrm{~cm} \\
\text { Ltubo } \approx 283 \mathrm{~cm}
\end{gathered}
$$

Debido a que el proceso a tratar se basa en reacciones exotérmicas, se genera una cantidad considerada de energía manifestada en forma de calor. Por esto, el reactor se debe diseñar para que el área de recibo sea la necesaria para disipar el calor.

$$
\begin{aligned}
& \text { Ltubo }=283 \mathrm{~cm} \\
& \begin{aligned}
\text { dtubo }=0.5 \mathrm{in}=1.27 \mathrm{~cm} \\
\boldsymbol{A}=\text { Ltubo } * \text { dtubo } * \pi \\
\boldsymbol{A}=\begin{array}{r}
283 \mathrm{~cm} * 1.27 \mathrm{~cm} * \pi \\
\boldsymbol{A}=0.1129 \mathrm{~m}^{2}
\end{array}
\end{aligned}
\end{aligned}
$$

\subsection{Código de programación en EES: modelo matemático}

Se describe el código utilizado en el programa EES para solucionar las ecuaciones del modelo matemático, y así compararlas con los resultados de la simulación [13].

Parámetros de entrada y suposiciones de funcionamiento: pérdida de calor insignificante alrededor del reactor; propiedades constantes; cambios de energía cinética y potencial insignificantes; factores de impureza insignificantes; condiciones de estado estable; condiciones de estado estable.
$T 1=273+23 ; \quad P 1=P$ o\#; $T 2=273+30 ; \quad Z_{-} 1=0 ; \quad V_{-} 1=0 ;$

$Z \_2=-0,5 ; D i=0,00848336 ; D o=0,0127 ; L=2,83$;

$K=k_{-}($'Aluminum'; $\quad 293)$; $\quad$ OMEGA $=10,472$;

r_rodete $=0,05$

$C V($ Ethanol $; T=T 1 ; P=P 1)=c v$

$C P($ Ethanol $; T=T 1 ; P=P 1)=$ cpet

$C P($ Water $; T=T 1 ; P=P 1)=$ cpagua

Visc_eth $=$ VISCOSITY $($ Ethanol $; T=318 ; P=P 1)$

$\operatorname{DENSITY}($ Ethanol $;=T 2 ; P=P 1)=$ Den_eth

DENSITY $($ Water $; T=280,5 ; P=P 1)=$ Den_wat

Visc_agua $=$ VISCOSITY $($ Water $; T=280,5 ; P=P 1)$

CONDUCTIVITY $($ Ethanol $; T=318 ; P=P 1)=K \_$eth

CONDUCTIVITY $($ Water $; T=280,5 ; P=P 1)=K_{-}$wat

$P R A N D T L($ Ethanol $; T=318 ; P=P 1)=p r_{-}$eth

PRANDTL $($ Water $; T=280,5 ; P=P 1)=p r \_$wat

GAMMA $=(g \# *$ DENSITY $($ Water $; T=T 1 ; P=P 1)) / 1000$

$A \_o=P I^{*} D o * L ; \quad \quad A \_i=P I^{*} D i * L ; \quad A \_i=A$;

A_serpentin $=(P I / 4) * D i^{\wedge} 2$

$m \_$Ethanol $=10 *(1 / 1000) * D E N S I T Y($ Ethanol $; T=318 ; P=$

P1)

q_ethanol $=m \_E t h a n o l *\left(\right.$ cpet $\left.^{*}((60+273)-(30+273))\right)$

"Interior del tubo (agua)"

"Aplicando la ecuacion de Bernoulli a todo el sistema"

$P 1 / G A M M A+\left(V_{-} l^{\wedge} 2 /(2 * g \#)\right)+Z_{-} l=(P 1 / G A M M A)+\left(V_{-}\right.$

$\left.2^{\wedge} 2 /(2 * g \#)\right)+Z \_2$

$m \_d o t=D E N S I T Y($ Water $; T=T 1 ; P=P 1) *\left(V \_2 * A \_\right.$serpent

in)

$R e \_i=\left(4 * m \_d o t\right) /\left(P I * D{ }^{*} \operatorname{VISCOSITY}(\right.$ Water $; T=280,5 ; P$

$=P 1)$ )

"Flujo turbulento"

"Para hallar el factor de friccion (f) tendremos en cuenta las siguientes restricciones: $0,5<P r_{-} a g u a<2000$

y $3000<R e \_i<5 * 10^{\wedge} 6$. "

$f=\left(0,790 * L N\left(R e_{-} i-1,649\right)\right) \wedge(-2)$

$N u r_{-} i=\left((f / 8) *\left(R e_{-} i-\right.\right.$

$1000) * P R A N D T L($ Water $; T=280,5 ; P=P 1)) /((1+(12,7 *(f$

18)^0,5)*(PRANDTL $\left.\left.\left.(\text { Water } ; T=280,5 ; P=P 1)^{\wedge}(2 / 3)-1\right)\right)\right)$

$N u r \_i=h \_i * D i / C O N D U C T I V I T Y($ Water $; T=280,5 ; P=P 1$

"Exterior del tubo (Ethanol)"

$V \_$eth $=O M E G A * r_{-}$rodete

$R e_{-} o=\left(D E N S I T Y(E\right.$ thanol $\left.;=318 ; P=P 1) * V \_e t h * D o\right) / V$

ISCOSITY (Ethanol;T=318;P=P1)

"Flujo turbulento"

$R e \_i * P R A N D T L($ Ethanol $; T=318 ; P=P 1)=$ Restriccion

"Como Re_i*Pr_Ethanol>0.2 Se usa la siguiente ecuacion para hallar el numero de Nusselt" 
Nur_o $=0,3+\left(0,62 * R e \_o^{\wedge}(1 / 2) *(P R A N D T L\right.$ (Ethanol $; T=$ $\left.318 ; P=P 1))^{\wedge}(1 / 3)\right) /(1+(0,4 /(P R A N D T L($ Ethanol $; T=318$ $\left.; P=P 1)))^{\wedge}(2 / 3)\right)^{\wedge}(1 / 4) *((1$

$\left.\left.+\left(R e_{-} o / 282000\right)^{\wedge}(5 / 8)\right)\right)^{\wedge}(4 / 5)$

Nur_o=h_o*Do/CONDUCTIVITY(Ethanol;T $=318 ; P=P$ 1)

$\left(1 / U^{*} A\right)=\left(1 / h_{-} i * A \_i\right)+\left(L N(D o / D i) /\left(2 * P I * K^{*} L\right)\right)+\left(1 / h_{-}\right.$ $\left.o * A \_o\right)$

"De la tabla 11-2 del libro Transferencia De Calor de Frank Incropera, podemos corroborar que el valor del U hallado para los fluidos en proceso esta en el rango de 250-700"

$L M T D=(((333-283))-(303-278)) / L N((333-283) /(303-$ 278))

q_water $=(U / 1000) * A *(L M T D)$

"Para conocer el tiempor en cual se lleva a cabo el proceso de enfriamiento tenemos:"

Tiempo $=\left(q \_\right.$ethanol/q_water $) / 60$

\subsection{Configuración de simulación: Flow Simulation}

Como fase inicial, se obtiene el modelo 3D del objeto de estudio. En la tabla 3 se encuentran los datos de la geometría del reactor y del intercambiador de calor. Se destaca la conductividad térmica de cada material, pues es una variable importante al momento de calcular la transferencia de calor por conducción [14].

Tabla 3. Datos de geométricos del reactor y del intercambiador de calor

\begin{tabular}{|c|c|c|}
\hline Características & Reactor & $\begin{array}{c}\text { Intercambiador de } \\
\text { calor }\end{array}$ \\
\hline Altura / longitud & $0.40 \mathrm{~m}$ & $2.83 \mathrm{~m}$ \\
\hline Diámetro & $0.20 \mathrm{~m}$ & $0.0127 \mathrm{~m}$ \\
\hline Espesor & $0.002 \mathrm{~m}$ & $0.00042164 \mathrm{~m}$ \\
\hline $\begin{array}{c}\text { Volumen / área de } \\
\text { disipación }\end{array}$ & $0.01251 \mathrm{~m}^{3}$ & $\begin{array}{c}0.1129 \mathrm{~m}^{2} \\
(6 \text { espiras })\end{array}$ \\
\hline Material & Acero & Aluminio \\
\hline $\begin{array}{c}\text { Conductividad } \\
\text { térmica a } 45^{\circ} \mathrm{C}\end{array}$ & $47-58 \mathrm{~W} / \mathrm{m} * \mathrm{~K}$ & $237 \mathrm{~W} / \mathrm{m} * \mathrm{~K}$ \\
\hline
\end{tabular}

Fuente: elaboración propia.

De acuerdo con los datos anteriores, en la figura 4 se realizó la esquematización del dispositivo en el software CAD Solidworks.

Una vez se define la geometría, esta se carga al módulo Flow Simulation, en el cual se establecen los parámetros de operación del reactor, como composición química de los reactivos y los reactantes; sus propiedades termofísicas, como densidad, calor específico, conductividad térmica, viscosidad dinámica y número de Prandtl [14], como se observan en la tabla 4.



Figura 4. Esquematización del dispositivo: cuerpo del reactor, dispositivo de agitación, intercambiador de calor. Fuente: elaboración propia.

Dentro del sistema global de experimento se definen los limites principales, donde el serpentín constituye el elemento principal tipo Inlet que reproduce la entrada de fluido al sistema, como lo hizo Villa-Salazar en las simulaciones realizadas en su proyecto. [15].

Flow Simulation tiene la función de pared modificada, con la que admite el modelo de transición laminarturbulento, además representa la compresibilidad de los flujos automáticamente. El módulo de simulación se basa en el modelo de turbulencia k- $\varepsilon$, que describe los flujos laminares, turbulentos y transicionales instintivamente, como lo explica Dassault Systemes, en su guía de usuario [16].

Tabla 4. Propiedades físico-químicas de los reactivos y reactantes

\begin{tabular}{|c|c|c|}
\hline Propiedades & $\begin{array}{c}\text { Datos alcohol } \\
\mathbf{T}=\mathbf{4 5}{ }^{\circ} \mathbf{C}\end{array}$ & $\begin{array}{c}\text { Datos agua } \\
\mathbf{T}=7.5{ }^{\circ} \mathbf{C}\end{array}$ \\
\hline $\begin{array}{c}\text { Temperatura de } \\
\text { entrada }\end{array}$ & $60{ }^{\circ} \mathrm{C}$ & $5{ }^{\circ} \mathrm{C}$ \\
\hline $\begin{array}{c}\text { Temperatura de } \\
\text { salida }\end{array}$ & $30^{\circ} \mathrm{C}$ & $10^{\circ} \mathrm{C}$ \\
\hline $\begin{array}{c}\text { Calor especifico } \\
\text { Conductividad } \\
\text { térmica }\end{array}$ & $2.48 \mathrm{KJ} / \mathrm{Kg} * \mathrm{~K}$ & $4.198 \mathrm{KJ} / \mathrm{Kg} * \mathrm{~K}$ \\
\hline Densidad $\rho$ & $781.4 \mathrm{~kg} / \mathrm{m}^{3}$ & $999.93 \mathrm{~kg} / \mathrm{m}^{3}$ \\
\hline $\begin{array}{c}\text { Viscosidad } \\
\text { dinámica } \mu\end{array}$ & $0.0007588 \mathrm{~Pa}-\mathrm{s}$ & $1413 \times 10^{-6} \mathrm{~Pa}-\mathrm{s}$ \\
\hline Prandtl & 13.06 & 10.53 \\
\hline
\end{tabular}

Fuente: [14]. 
Análisis térmico, modelamiento matemático y simulación de un reactor de agitación discontinuo para volumen específico

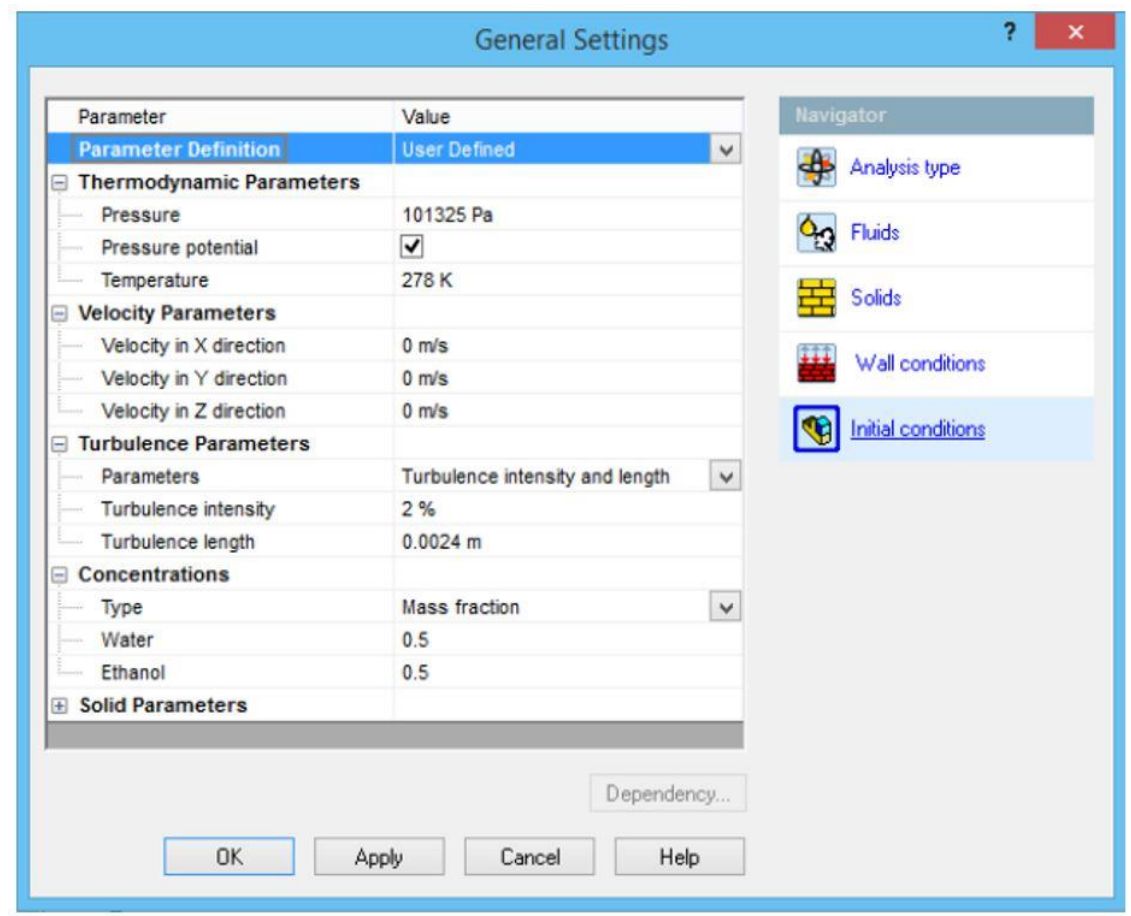

Figura 5. Cuadro de diálogo, configuración del sistema. Fuente: Solidworks 2016.

En un principio, se delimita el volumen de control, y se inicia el módulo de ayudante de simulación Wizard, seleccionando el sistema de unidades y los mecanismos de transferencia de calor. Como se ve en las figuras $5 \mathrm{y}$ 6 , se agrega el régimen de turbulencia al objeto de estudio, y se definen los fluidos que intervienen en la simulación. También se considera la especificación de las variables de presión y el flujo del fluido dentro del sistema [17], [18].

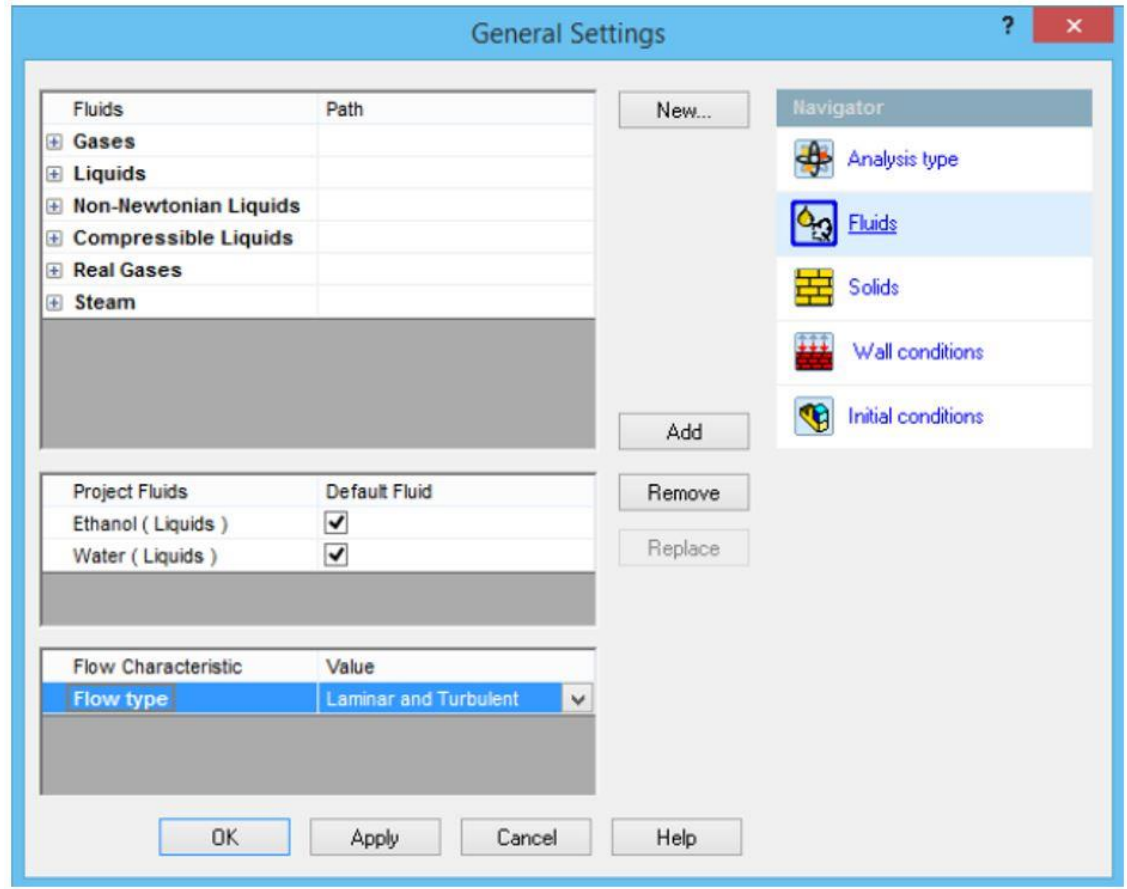

Figura 6. Cuadro de diálogo, configuración de los fluidos de trabajo. Fuente: Solidworks 2016. 


\section{Resultados}

\subsection{Modelo matemático}

En la figura 7 se muestra el comportamiento del reactor de agitación, específicamente cómo la temperatura de operación dentro del equipo disminuye en función del tiempo de operación.



Figura 7. Relación temperatura en función del tiempo. Fuente: elaboración propia.

De acuerdo con la gráfica, una vez el dispositivo entra en su régimen de trabajo ideal, se tiende a disipar más rápido el calor dentro del tanque. Al iniciar la reacción química, se genera un incremento súbito de temperatura, pues el reactor viene de un estado de reposo actual.

\subsection{Simulación}

En la fase de análisis de datos se deben seleccionar principalmente las variables que se quieren evaluar. En la figura 8 se muestra cómo se configura esta opción de posprocesamiento desde el módulo de Flow Simulation, y se establece un tiempo para observar los resultados en un instante específico.

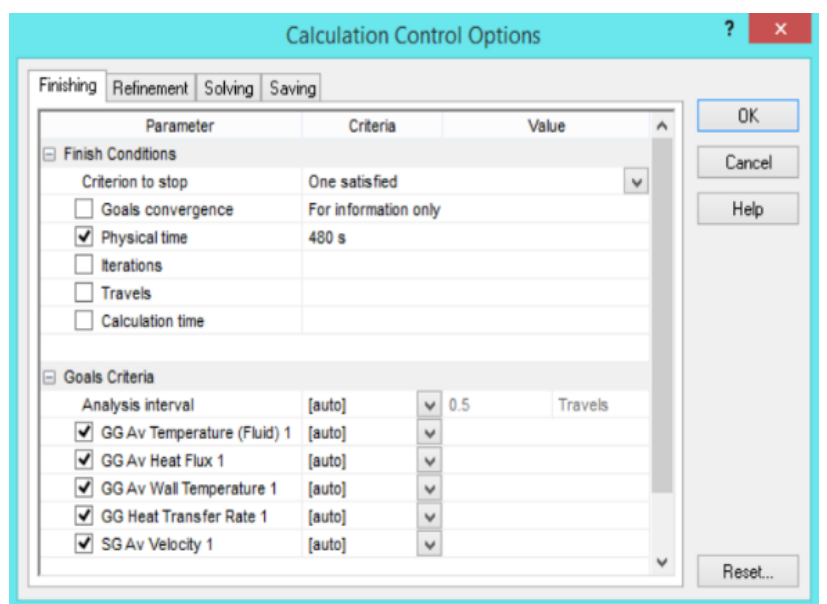

Figura 8. Parámetros de posprocesamiento. Fuente: Solidworks 2016.
La simulación muestra los resultados de la temperatura dentro del serpentín del reactor en la figura 9.



Figura 9. Temperatura en el serpentín del reactor. Fuente: elaboración propia.

Además, en la figura 10 se ilustra cómo afecta el calor que disipa el serpentín a todo el cuerpo del reactor. Se observa la temperatura de todo el equipo en 3 instantes de operación: en la fase de inicio, a los 240 segundos de operación y a los 480 segundos.

\section{Conclusiones}

Se evidencia un buen desempeño por parte del reactor de agitación discontinuo, además se demuestra que es el dispositivo adecuado para lograr una mezcla óptima de acuerdo con el producto final que se quiere obtener.

El modelo matemático y la simulación presentan resultados similares, específicamente en el comportamiento de la temperatura a lo largo del ciclo de operación del reactor. En la fase inicial se alcanza un pico de $333 \mathrm{~K}$ dentro del dispositivo. Conforme pasa el tiempo, la temperatura disminuye constantemente. Para ser precisos, a los 280 segundos está por debajo de los $240 \mathrm{~K}$, y a los 480 segundos ha descendido hasta el rango de $305 \mathrm{~K}$. 


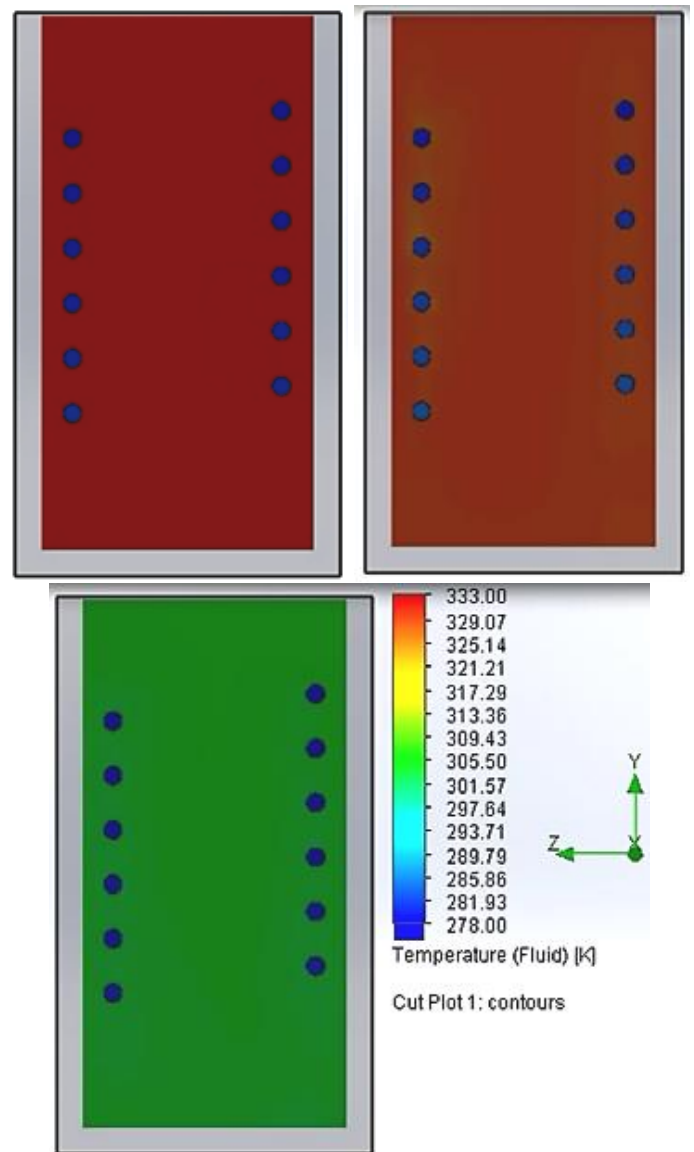

Figura 10. Temperatura dentro del reactor: Fase inicial, 240 s de operación, 480 s de operación respectivamente. Fuente: elaboración propia.

El desempeño del intercambiador de calor es el adecuado, pues en la base del cilindro no se superan los $280 \mathrm{~K}$, rango apropiado para que los fluidos de operación puedan reaccionar correctamente en las fases adecuadas y no rompan sus enlaces químicos antes de tiempo.

Este método es el indicado para diseñar reactores de agitación, pues se logró una metodología matemática, partiendo de premisas prácticas o experimentales y validadas a través de software asistido por computadora.

\section{Agradecimientos}

El grupo de trabajo desea agradecer al departamento de Fluidos y Térmicas de la Universidad Francisco de Paula Santander, por poner a nuestra disposición el laboratorio de plantas térmicas. Además, se hace una mención especial de los alumnos Andrés Báez, Leopoldo Valero y Marcos Duarte, del programa de Ingeniería Mecánica, por su cooperación en la elaboración de las pruebas y en el posterior análisis de datos.

\section{Referencias}

[1] G. Castellar, E. R. Angulo, B. M. Cardozo, "Transesterification vegetable oils using Heterogeneous catalysts," Prospect, vol 12, núm. 2, pp. 90-104, 2014. doi: $10.15665 /$ rp.v12i2.293

[2] T. Leevijit, C. Tongurai, G. Prateepchaikul, W. Wisutmethangoon, "Performance test of a 6-stage continuous reactor for palm methyl ester product ion," Bioresource Technology, vol. 99, núm. 1, pp. 214-221, 2008. doi 10.1016/j.biortech.2006.11.052

[3] S. D. Romano, E. González-Suárez, M. A. Laborde, Combustibles Alternativos. Buenos Aires, Argentina: Ediciones Cooperativas, 2005.

[4] M. Fangrui, M. Hanna, "Biodiesel production: A Review," Bioresource Technology, vol. 70, pp. 1-15, núm. 1, 1999. doi: 10.1016/S0960-8524(99)00025-5 
[5] S. Furuta, H. Matsuhashi, K. Arata, "Biodiesel fuel production with solid amorphous - zirconia catalysis in fixed bed reactor," Biomass and Bioenergy, vol. 30, núm. 10, pp. $870-873,2006$. doi: 10.1016/j.biombioe.2005.10.010

[6] W. G. Morales, P. Dagnino, A. Diaz, N. Polich, A. Sequeira, E. Chamorro, "Proceso semicontínuo en la transesterificación de triglicéridos de semilla de algodón”, Grupo de Investigación en Química Orgánica Biológica (Quimobi) [En línea]. Disponible en: https://www.researchgate.net/publication/267234769

[7] K.G. Denbigh, J.C. Turner, Introducción a la teoría de los reactores químicos. México: Editorial Limusa, 1990.

[8] M. Bayas, C. Nuñez - Aguagallo, "Diseño y construcción de un reactor continuo de mezcla completa", Trabajo de grado, Escuela Superior Politécnica de Chimborazo, 2011 [En línea]. Disponible en: http://dspace.espoch.edu.ec/handle/123456789/1968

[9] M. Dolors-Grau, L. Puigjaner-Corbella, "Estudio del comportamiento de reactores discontinuos y semicontinuos: modelización y comprobación experimental", Universitat Politècnica De Catalunya, 1999. [En línea]. Disponible en: http://www.tdx.cat/bitstream/handle/10803/6463/02_gra uVilalta_capitol_1.pdf;jsessionid=D7342C7FADDC5E 1B0DBD4090299A32D7? sequence $=2$

[10] C. Hernández-Pedrera, M. Rivera-Soto, R. MatosDurán, Y. Piñó-Cuenca, "Estudio de sensibilidad paramétrica en reactores continuos con agitación", Revista Tecnología Química, vol. 34, no. 1, 2014. doi: 10.1590/2224-6185.2014.1.\%25x

[11] W. McCabe, J. Smith, P. Harriott, Operaciones unitarias en ingeniería química. México: Editorial McGraw Hill, 2007.

[12] D. Kern, Procesos de transferencia de calor. Mexico: Mc Graw Hill Book, 1999.

[13] S. A. Klein, "Handbook Engineering Equation Solver EES," F-Chart Software, 2016.

[14] F. Incropera, D. P. DeWitt, Fundamentos de transferencia de calor. Pearson Educación, 1999.

[15] D. Villa, D. Hincapié, y E. Torres, "Simulación computacional de la transferencia de calor en herramientas usadas en soldadura por fricción- agitación," Rev. UIS Ing., vol. 14, núm. 2, pp. 19-26, 2015. doi: 10.18273/revuin.v14n2-2015003

[16] Guia de usuario Solidworks Flow Simulation, Dassault Systèmes - SolidWorks Corporation, 2016.

[17] G. Valle-Tamayo, L. Valbuena-Luna, C. RojasBeltrán, M. Cabarcas-Simancas, "Modelo numérico para el análisisy el diseño de redes de tuberías para flujo bifásico," Rev. UIS Ing., vol. 17, núm. 2, pp. 201-214, 2018. doi: 10.18273/revuin.v17n2-2018018

[18] J. Matsson, An introduction to SolidWorks Flow Simulation. U.S.A: Schroff Development Corp, 2013. 